\title{
Radiologic Findings of Complicated Alloplastic Implants in the Nasal Dorsum
}

\author{
Sung Hee $\mathrm{Kim}^{1}$ (D) $\cdot$ Ji Won Kim ${ }^{1}$ (D) $\cdot$ Yong Ju Jang ${ }^{2}$ (D) \\ ${ }^{1}$ Department of Otorhinolaryngology-Head and Neck Surgery, National Medical Center, Seoul; \\ ${ }^{2}$ Department of Otolaryngology, Asan Medical Center, University of Ulsan College of Medicine, Seoul, Korea
}

Objectives. When performing cosmetic rhinoplasty with alloplastic materials, complications such as implant visualization, inflammation, dislocation, and extrusion should be thoroughly evaluated. Although computed tomography (CT) can provide useful information about the implant status and its interaction with the skin soft tissue envelope (SSTE), the radiologic findings of these interactions have rarely been reported.

Methods. We retrospectively reviewed the data of 80 patients who underwent facial bone CT or ostiomeatal unit CT at Asan Medical Center between July 2008 and January 2020 for the evaluation of dorsal implants with complications. We reviewed the implantation period, implant dislocation, implant curling or deformation, radiodensity (in Hounsfield units), and nasal bone changes including bone erosion or hyperostosis.

Results. Of the 80 patients, 67 (83.8\%) had silicone implants and 13 (16.2\%) had Gore-Tex implants. The radiologic findings of the silicone implants were as follows: maintenance of the implant shape $(80.6 \%)$, radiolucency (similar density to that of fat tissue) halo (83.6\%), and homogeneous attenuation (82.1\%). Peri-implant calcification was often found in silicone implants with $>20$-year implantation periods. The findings of Gore-Tex were as follows: curling or deformation $(84.6 \%)$, heterogeneous attenuation $(84.6 \%)$, and consistent peri-implant calcification over time.

Conclusion. Silicone and Gore-Tex implants have distinctive radiologic features. These findings of alloplastic materials help us to understand how implants behave in the nasal dorsum and how they affect the SSTE.

Keywords. Silicone; Polytetrafluoroethylene; Radiology; Rhinoplasty; Computed Tomography

\section{INTRODUCTION}

Silicone and expanded polytetrafluoroethylene (Gore-Tex) are commonly used materials for dorsal implantation in rhinoplasty. They are easy to handle, yield desirable cosmetic outcomes, and can prevent donor-site morbidity and implant resorption as seen with autologous implants [1]. However, some surgeons are unwilling to use these implants because of concerns regarding the incidence of implant infection, calcification, displacement, and extrusion [2].

- Received August 15, 2020

Revised December 8, 2020

Accepted December 9, 2020

- Corresponding author: Yong Ju Jang

Department of Otolaryngology, Asan Medical Center, University of Ulsan College of Medicine, 88 Olympic-ro 43-gil, Songpa-gu, Seoul 05505, Korea Tel: +82-2-3010-3712, Fax: +82-2-489-2773

E-mail: jangyj@amc.seoul.kr
The effects of alloplastic implants on the surrounding tissues and their long-term associated changes have been studied using ultrasound and histologic examinations [3-5]. The implantation of silicone can induce a macrophage-related foreign body reaction, which can surround the implant with fibrous tissue; this tissue is defined as the implant capsule. In dorsal augmentation using Gore-Tex, tissue ingrowth into the implant is a common histologic finding, which makes it difficult to remove the implant from the tissue during revision surgery [6]. The thickness of a dorsal alloplastic implant and its changes over time have been studied using ultrasonography. The postoperative thickness of Gore-Tex implants decreased by $29 \%$, and there was no significant correlation between the degree of change in implant thickness and the length of time after surgery [5].

When complications are observed in dorsal alloplastic implants, it is mandatory to consider the removal of the implant. In this situation, radiologic evaluations can provide useful infor-

Copyright (C) 2021 by Korean Society of Otorhinolaryngology-Head and Neck Surgery.

This is an open-access article distributed under the terms of the Creative Commons Attribution Non-Commercial License (https://creativecommons.org/licenses/by-nc/4.0)

which permits unrestricted non-commercial use, distribution, and reproduction in any medium, provided the original work is properly cited. 
mation about the type and status of the implant and its interactions with the surrounding tissues. Even without opening the nose, it is possible to visualize the events taking place inside of the nasal skin soft tissue envelope (SSTE). At our clinic, computed tomography (CT) scans would not normally be performed after rhinoplasty; however, radiologic images are often taken for treatment planning in patients with complications of dorsal implants. However, to date, studies on the radiologic findings of dorsal implants and changes in implant shape and the effects on surrounding tissue have rarely been reported. Here, we present radiologic findings of implant changes in the SSTE of the nasal dorsum and its interactions with the surrounding tissues.

\section{MATERIALS AND METHODS}

\section{Study population}

We retrospectively reviewed the data on patients who underwent facial bone CT or ostiomeatal unit (OMU) CT at the Asan Medical Center between July 2008 and January 2020 for preoperative evaluation of complicated dorsal implants. This study was approved by the Institutional Review Board of the Asan Medical Center and the requirement for individual patient written informed consent was waived. Eighty patients who had complicated dorsal alloplastic implants were included in this study, and majority of the patients had silicone or Gore-Tex implants. We excluded patients (1) who had other types of alloplastic implants, (2) who had no CT sagittal plane images, (3) with lack of medical records. To find out the interaction between the dorsal implant and surrounding tissue, we reviewed the implantation period, dislocation, implant curling, or deformation, Hounsfield unit (HU), and the changes occurring at the nasal bone, including bone erosion or hyperostosis.

\section{Radiologic findings}

The radiologic findings of each implant were reviewed by a head and neck radiology specialist. HU was measured on the axial plane of the $\mathrm{CT}$ at the widest point of the implant to check it at the same point each. Heterogeneity was evaluated consid-

\section{H I G H L I G G H T T S}

- Silicone dorsal implants mostly maintain their shape inside the skin soft tissue envelope.

- Implant curling or deformation was frequently observed with Gore-Tex implants.

- Silicone implants showed distinctive peri-implant radiolucent halos.

- Calcification was often found in silicone implants with $>20$ year implantation periods. ering whether there was a low-density or high-density area inside of the implant compared to that of the entire implant. The implant margin was either classified as a smooth regular margin or as an irregular deformed-looking margin. Nasal bone changes were evaluated in terms of erosion or hyperostotic lesions. Lastly, surrounding soft tissue changes were evaluated on the sagittal plane of the CT.

\section{RESULTS}

Table 1 shows the characteristics of the patients according to the type of alloplastic implant. Eighty patients (including 51 women) with a mean age of 39.6 years (range, 19-73 years) were enrolled. Sixty-seven patients had silicone implants, and 13 had Gore-Tex implants. About $50 \%$ of the patients had an implantation period of more than 10 years. Table 2 summarizes the complication patterns in patients who underwent revision surgery. Common complications associated with silicone implants included short nose deformity $(20.9 \%)$ and implant displacement/ deviation (19.4\%), and those associated with Gore-Tex implants included implant displacement/deviation (23\%) and dorsal irregularity $(23 \%)$. Esthetic dissatisfaction most frequently occurred because of an unnatural-looking nasal shape and nostril asymmetry.

Facial bone or OMU CT revealed important findings of dorsal alloplastic implants. Table 3 shows the radiologic findings associated with each material. Implant curling or deformation was more common in patients with Gore-Tex implants (84.6\%) than in those with silicone implants (19.4\%) (Fig. 1). Implant displacement was observed more frequently in patients with silicone implants $(40.3 \%)$ than in those with Gore-Tex implants (23\%). We detected a peri-implant radiolucent halo, which refers to a low CT-attenuated region similar to that in fat tissue (Fig. 2). This halo was present in the radiologic images of most patients who underwent rhinoplasty with a silicone implant. We also frequently detected intra-implant low CT-attenuated regions, which were found in more than $80 \%$ of patients with Gore-Tex implants (Fig. 3). Implant-associated secondary bony

Table 1. Comparison of the characteristics of the patients and implant insertion periods according to the alloplastic implant material used

\begin{tabular}{lcc}
\hline Variable & Silicone $(n=67)$ & Gore-Tex $(n=13)$ \\
\hline Sex $($ M:F) & $21(31.4): 46(68.6)$ & $8(61.5): 5(38.5)$ \\
Mean age (yr) & 37.9 & 41.4 \\
Mean implantation period $(\mathrm{yr})$ & & \\
$<10$ & $35(52.2)$ & $6(46.2)$ \\
10 to $<20$ & $26(38.8)$ & $4(30.8)$ \\
20 to $<30$ & $5(7.5)$ & $3(23)$ \\
$\geq 30$ & $1(1.5)$ & 0 \\
\hline
\end{tabular}

Values are presented as number (\%). 
changes such as bone resorption and hyperostosis mainly occurred in patients with Gore-Tex implants who had an implantation period of more than 10 years (Fig. 4). Peri-implant calcifica-

Table 2. Complication patterns in patients who underwent revision surgery

\begin{tabular}{lcc}
\hline Complication pattern before surgery & Silicone $(\mathrm{n}=67)$ & Gore-Tex $(\mathrm{n}=13)$ \\
\hline Implant displacement/deviation & $13(19.4)$ & $3(23)$ \\
Short nose & $14(20.9)$ & - \\
Infection & $8(11.9)$ & $1(7.7)$ \\
Recurrent inflammation & $8(11.9)$ & $2(15.4)$ \\
Extrusion & $3(4.5)$ & - \\
Trauma & $3(4.5)$ & $1(7.7)$ \\
Low radix & $2(2.9)$ & $2(15.4)$ \\
Nasal discomfort & $4(5.9)$ & - \\
Dorsal irregularity & $2(2.9)$ & $3(23)$ \\
Aesthetic dissatisfaction & $10(14.9)$ & $1(7.7)$
\end{tabular}

Values are presented as number (\%).
Table 3. Comparison of the radiologic findings according to the alloplastic implant material used

\begin{tabular}{lcc}
\hline Radiologic finding & Silicone $(\mathrm{n}=67)$ & Gore-Tex $(\mathrm{n}=13)$ \\
\hline Curling of deformation & $13(19.4)$ & $11(84.6)$ \\
Displacement & $27(40.3)$ & $3(23)$ \\
Horizontal & $23(85.2)$ & $2(66.6)$ \\
Vertical & $4(14.8)$ & $1(33.3)$ \\
Peri-implant radiolucent halo & $56(83.6)$ & $3(23)$ \\
Intra-implant low attenuation & $12(17.9)$ & $11(84.6)$ \\
Secondary bony change & & \\
No change & $53(79.1)$ & $5(38.5)$ \\
Bone resorption & $8(11.9)$ & $4(30.7)$ \\
Hyperostosis & $6(9.0)$ & $4(30.7)$ \\
Peri-implant calcification (calcification:total) $(\mathrm{yr})$ & \\
$<10$ & $2 / 36$ & $2 / 6$ \\
10 to $<20$ & $4 / 25$ & $2 / 4$ \\
20 to $<30$ & $2 / 5$ & $2 / 3$ \\
$\geq 30$ & $1 / 1$ & - \\
\hline
\end{tabular}

Values are presented as number (\%).
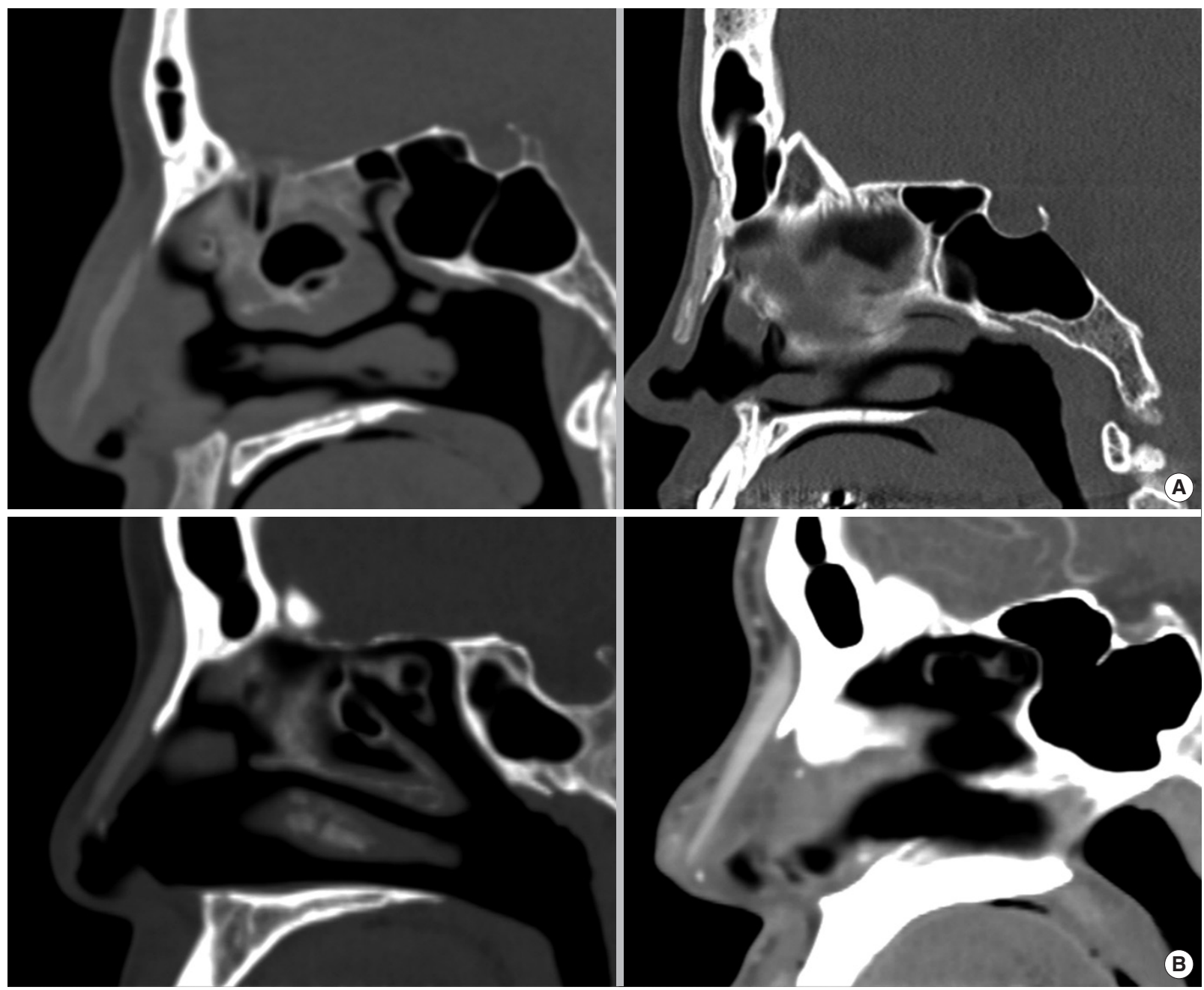

Fig. 1. Shapes of the dorsal alloplastic implants. Gore-Tex implants showed implant curling and deformation in the sagittal plane on computed tomography $(A)$, while silicone implants usually maintained their shape inside the skin soft tissue envelope (B). 


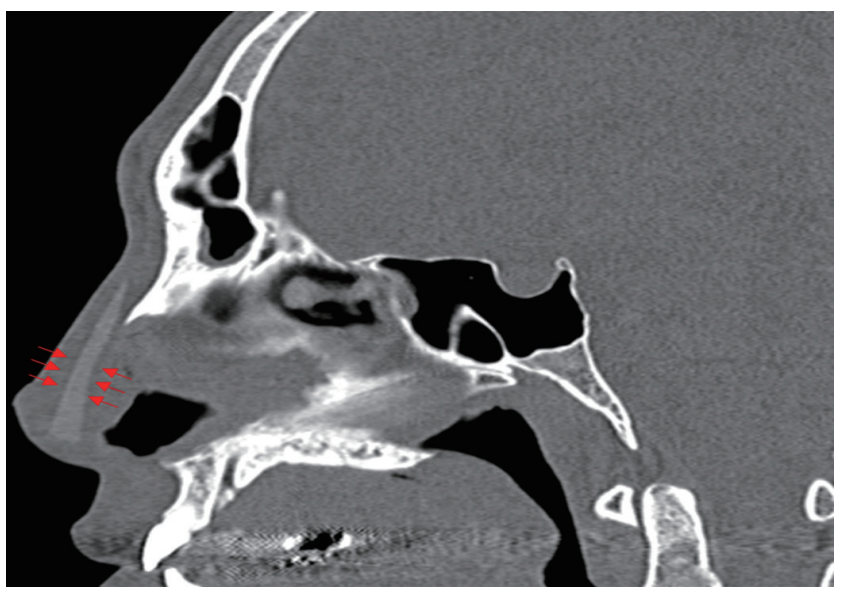

Fig. 2. The peri-implant radiolucent halo found with silicone implants (red arrows).

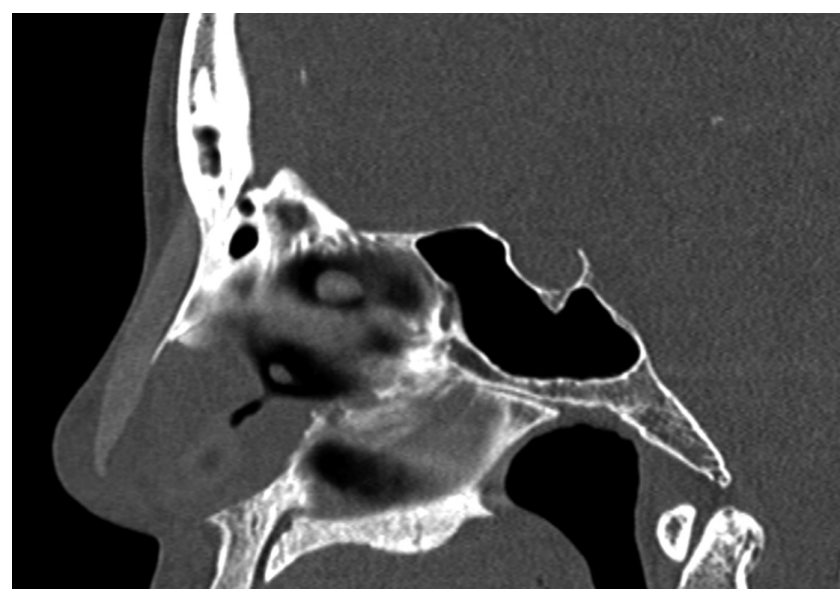

Fig. 3. The intra-implant low attenuation found in Gore-Tex implants.
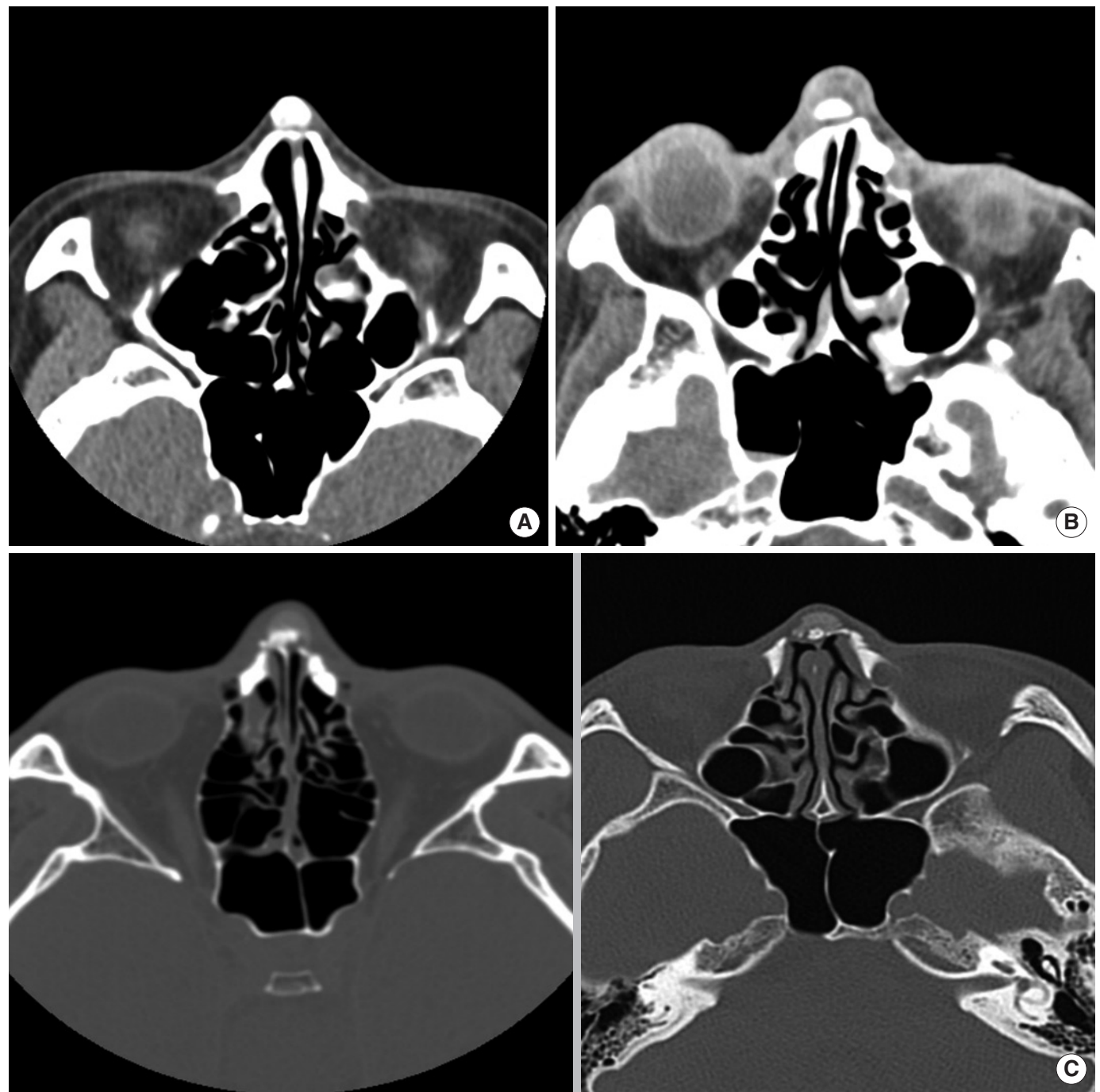

Fig. 4. Erosion of the nasal bone was observed due to (A) a silicone implant (the period of insertion was 25 years), (B) a Gore-Tex implant (the period of insertion was 14 years). (C) Hyperostosis of the nasal bone was observed due to a Gore-Tex implant; the periods of insertion were 4 years (left), and 14 years (right). 

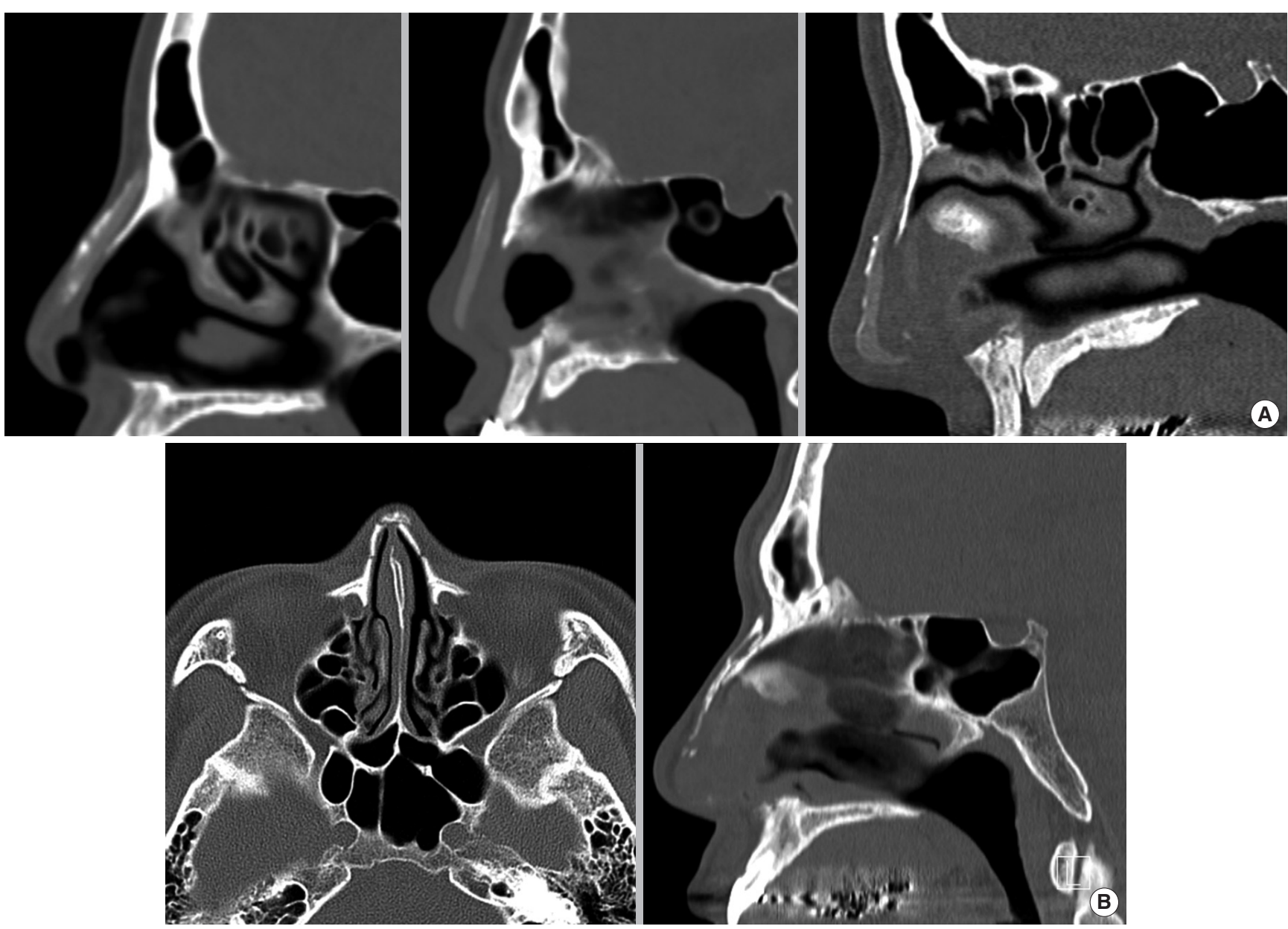

Fig. 5. Peri-implant calcifications of dorsal alloplastic implants. (A) Silicone implants, with periods of insertion of 25 years (left), 40 years (middle), and 18 years (right), (B) Gore-Tex implants (the period of insertion was 15 years).

tion was observed in patients with both types of implants. In patients with silicone implants, calcification was associated with the implantation period. Implant calcification was found in $50 \%$ of the patients with Gore-Tex implants, but this was not related to the implantation period (Fig. 5).

\section{DISCUSSION}

The most common type of rhinoplasty performed on Asians is augmentation rhinoplasty, and various alloplastic materials are used for tissue augmentation. Silicone is the most common implant material for dorsal augmentation owing to its convenience and relative hardness, which make it suitable for shaping the nose in Asian patients with thick skin [7]. Gore-Tex is also widely used due to its advantages, which include low tissue reactivity, excellent biocompatibility, reasonable cost, and microporous composition, which encourages tissue ingrowth and thereby promotes implant stability [8]. In the present study, there were distinct radiologic differences between silicone and Gore-Tex im- plants in the nasal dorsum in terms of their shape, attenuation, and interaction with the surrounding tissues.

In particular, we found that silicone implants usually maintained their shape inside the SSTE (80.6\%), whereas Gore-Tex implants commonly exhibited curling or deformation $(84.6 \%)$. This could be attributed to the natural characteristics of GoreTex, a material that is stretchable and compressible to a certain degree. In Asians, the septal cartilage is relatively small, and the anterior septal angle is posteriorly located and weakly projected. Therefore, Gore-Tex tends to bend along this natural convexity of the dorsum with time.

Previous studies have reviewed the histologic findings of alloplastic implants, such as fibrous capsule formation, tissue ingrowth, microscopic calcification, and foreign body reactions [6,9-12]. It is noteworthy that some of these findings corroborate our study results. First, the fibrous capsule of silicone implants showed a peri-implant radiolucent halo (similar density to that of fat tissue) in our study. This radiologic finding was predominantly observed in patients with silicone implants. Second, tissue ingrowth into Gore-Tex implants was radiologically ob- 
served with intra-implant low attenuation; this finding was predominantly observed in patients with Gore-Tex implants. Third, peri-implant calcification was commonly observed in patients with silicone implants who had an implantation period of more than 20 years. In a histologic study, Jung et al. [10] showed that longer durations of silicone implantation led to more and broader calcifications, as observed with the naked eye on gross examination. These results were in agreement with our findings.

The radiologic finding of a peri-implant radiolucent halo relates to the capsule of the silicone implants, and that of intra-implant low attenuation indicates tissue ingrowth into the micropores of the Gore-Tex implants. The clinical importance of these findings is as follows. First, we can easily estimate the approximate thickness of the capsule around silicone implants. Second, based on the degree of heterogeneous attenuation, it is possible to predict the difficulty of dissection of Gore-Tex implants. Third, these findings allow surgeons to straightforwardly identify which implant was used in previous surgical procedures without opening the nose.

Few studies have evaluated the radiologic findings of alloplastic implants in the nasal dorsum [13,14]. Schatz et al. [13,14] reviewed the imaging findings of cosmetic facial implants and grafts. They presented the imaging findings of various alloplastic implants made of different materials, such as silicone rubber, Gore-Tex, polytetrafluoroethylene (Teflon), porous polyethylene (Medpor), and hydroxyapatite. However, unlike in our study, they did not describe the common imaging characteristics of specific alloplastic implants. In our analysis, we found distinct radiologic characteristics of nasal dorsal implants and evaluated their long-term changes. These findings are important because they can be used to determine the type of implant that was used to augment a patient's nasal dorsum before exposing the SSTE through surgery. Our study results also provide valuable information regarding preoperative evaluations, indicating what may eventually happen to the implants and surrounding tissue.

This study has some limitations. First, only two alloplastic materials - silicone and Gore-Tex-were reviewed and their distinctive imaging features were compared. Second, we used two different types of CT, which may have influenced attenuation. Third, patients' history of previous surgical procedures such as rasping or osteotomy could affect osteogenesis of the nasal bone. In future studies, we will need to unify the type of CT used for examinations and compare the imaging findings of various dorsal implants, as well as reviewing previous surgical records.

In this study, we observed various distinct radiologic characteristics of alloplastic materials, with a particular focus on silicone and Gore-Tex. Further studies including other types of alloplastic implants may provide additional information about the distinct radiologic characteristics of each material. Based on the present results, we conclude that the radiologic findings of alloplastic materials can help predict the behavior of implants in the nasal dorsum and their effects on SSTE.

\section{CONFLICT OF INTEREST}

No potential conflict of interest relevant to this article was reported.

\section{ORCID}

Sung Hee Kim https://orcid.org/0000-0001-7230-8774

JiWon Kim https://orcid.org/0000-0002-0882-9177

Yong Ju Jang https://orcid.org/0000-0001-7631-0388

\section{AUTHOR CONTRIBUTIONS}

Conceptualization: YJJ. Data curation: SHK, JWK. Formal analysis: SHK. Writing-original draft: SHK. Writing-review \& editing: SHK, YJJ.

\section{REFERENCES}

1. Na HG, Jang YJ. Dorsal augmentation using alloplastic implants. Facial Plast Surg. 2017 Apr;33(2):189-94.

2. Kim IS. Augmentation rhinoplasty using silicone implants. Facial Plast Surg Clin North Am. 2018 Aug;26(3):285-93.

3. Byun JW, Han JY, Ki SH, Lee SH, Shin J, Choi GS. Foreign body type nasal pseudocyst after augmentation rhinoplasty: histopathologically mucin-containing pseudocyst. Am J Dermatopathol. 2017 Jul;39(7):554-6.

4. SunwooW, Jung H, Kim DW, Jin HR. Immunohistochemical analysis of capsular contracture in silicone implant rhinoplasty. JAMA Facial Plast Surg. 2017 Sep;19(5):436-7.

5. Jung YG, Kim HY, Dhong HJ, Park KN, Lee HJ, Lim YJ, et al. Ultrasonographic monitoring of implant thickness after augmentation rhinoplasty with expanded polytetrafluoroethylene. Am J Rhinol Allergy. 2009 Jan-Feb;23(1):105-10.

6. Jang TY, Choi JY, Jung DH, Park HJ, Lim SC. Histologic study of Gore-Tex removed after rhinoplasty. Laryngoscope. 2009 Apr;119(4): 620-7.

7. Wang JH, Lee BJ, Jang YJ. Use of silicone sheets for dorsal augmentation in rhinoplasty for Asian noses. Acta Otolaryngol Suppl. 2007 Oct;(558):115-20.

8. Godin MS, Waldman SR, Johnson CM Jr. Nasal augmentation using Gore-Tex: a 10-year experience. Arch Facial Plast Surg. 1999 AprJun;1(2):118-21.

9. Kim HS, Park SS, Kim MH, Kim MS, Kim SK, Lee KC. Problems associated with alloplastic materials in rhinoplasty. Yonsei Med J. 2014 Nov;55(6):1617-23.

10. Jung DH, Kim BR, Choi JY, Rho YS, Park HJ, Han WW. Gross and pathologic analysis of long-term silicone implants inserted into the human body for augmentation rhinoplasty: 221 revision cases. Plast Reconstr Surg. 2007 Dec;120(7):1997-2003.

11. Moon KC, Lee KI, Lee JS, Kim AR, Dhong ES, Kim DW, et al. Lateonset inflammation in Asian rhinoplasty using alloplastic implants. 
Aesthetic Plast Surg. 2021 Apr;45:670-8.

12. Jang YJ, Moon BJ. State of the art in augmentation rhinoplasty: implant or graft? Curr Opin Otolaryngol Head Neck Surg. 2012 Aug; 20(4):280-6.
13. Schatz CJ, Ginat DT. Imaging features of rhinoplasty. AJNR Am J Neuroradiol. 2014 Feb;35(2):216-22.

14. Schatz CJ, Ginat DT. Imaging of cosmetic facial implants and grafts. AJNR Am J Neuroradiol. 2013 Sep;34(9):1674-81. 